\title{
Mythes et démystification dans le roman policier de Fred Vargas
}

Laetitia Gonon

\section{OpenEdition}

1 Journals

Édition électronique

URL : http://journals.openedition.org/recherchestravaux/434

DOI : 10.4000/recherchestravaux.434

ISSN : 1969-6434

Éditeur

UGA Éditions/Université Grenoble Alpes

\section{Édition imprimée}

Date de publication : 20 décembre 2010

Pagination : 119-135

ISBN : 978-2-84310-187-8

ISSN : 0151-1874

\section{Référence électronique}

Laetitia Gonon, "Mythes et démystification dans le roman policier de Fred Vargas », Recherches \& Travaux [En ligne], 77 | 2010, mis en ligne le 20 août 2012, consulté le 08 septembre 2020. URL : http:// journals.openedition.org/recherchestravaux/434; DOI : https://doi.org/10.4000/recherchestravaux 434 


\section{Mythes et démystification dans le roman policier de Fred Vargas}

Puisqu'il est ici question de mythe, peut-être faut-il commencer avec l'un d'eux : qui ignore aujourd'hui cette classification des librairies et bibliothèques qui sépare le rayon Littérature des rayons dits spécialisés de la littérature de science-fiction, la littérature policière, ou la littérature pour enfants? Cette classification donne l'impression que le mot littérature, lorsqu'il est suivi d'un complément, perd sa majuscule, comme si la Littérature s'imposait d'ellemême, au-delà des catégories, par le simple constat de son effort au style, ou de sa littérarité, et que ce seul trait commun à des œuvres diverses suffisait à les ranger sur les mêmes rayonnages. Ainsi la catégorisation commerciale en littérature policière - car « $[\mathrm{n}] \mathrm{ul}$ doute que nous soyons, avec la lecture policière, dans la littérature de marchér ${ }^{\prime}$-, littérature de science-fiction, littérature pour enfants, serait le signe de son infériorité (consentie par son classement), l'indice que là se trouve le dernier refuge des caricatures - des mythes, en somme.

On ne se saisit qu'avec précaution de ce terme de «mythe», dont la signification chez Barthes même reste fluctuante : il suffira de renvoyer ici à l'article de Serge Zenkine pour s'en convaincre. "Le mythe, aujourd'hui» redouble effectivement d'attributs pour définir son objet, comme "parole", "système de communication", "message», "parole volée et rendue», "vol de langage ». Nous voudrions conserver dans le sens que nous prêterons au terme cette dimension interlocutive (le mythe se construit surtout autour de ses lecteurs et de leurs représentations), mais lui ôter de sa complexité en l'utilisant simplement pour désigner, du roman policier, ce qui s'est figé en règles si bien connues et éprouvées qu'elles ont constitué un code, une histoire même,

I. J. Dubois, Le Roman policier ou la modernité, Nathan, I992, p. 47.

2. R. Barthes, Mythologies, Seuil, coll. «Points», 1957, p. I8I, 198 et 204. 
partagée par ses amateurs : le mythe veut que la première histoire policière soit celle d'EEdipe qui, à la recherche de l'assassin, ne se doute pas qu'il le connaît déjà; toujours selon le mythe, le roman policier trouve son origine dans la déduction modèle construite par le Zadig de Voltaire, a été initié par Edgar Allan Poe, et repris par Arthur Conan Doyle, Gaston Leroux et Agatha Christie 3 . Il s'agit bien ici, uniquement, "du roman policier classique [...], et qu'on peut appeler roman à énigme ${ }^{4} »$, celui qu'on a nommé par ailleurs whodunit (qui l'a fait?) : la question résume le récit à la révélation, à la fin de l'enquête, du nom du coupable. Car l'un des mythes du genre est indéniablement l'aspect ludique de la narration : cherchez à qui profite le crime, trouvez le coupable. Cet aspect ludique est sous-tendu, contrairement au roman noir, par la curiosité et non par le suspenses, si bien que le lecteur n'a pas à s'inquiéter pour les héros, "immunisés» contre le danger ${ }^{6}$. "Rien ne peut leur arriver ${ }^{7}$ " car ils doivent rester en vie pour assurer la prochaine enquête. Débarrassé du suspense potentiellement anxiogène, le lecteur se fait enquêteur, et ce dernier est lui-même souvent réduit à sa capacité de déduction et à ses "petites cellules grises" : personnage volontairement désincarné, le détective peut ne même pas quitter sa chambre pour résoudre le mystère, comme le lecteur ne quitte pas son fauteuil pour finir le livre.

Le roman policier présenterait ainsi le mythe d'une énigme à résoudre, d'une solution à trouver, dans une narration sans complexe, débarrassée de tout soupçon et de toute réflexion métalittéraire : Jacques Dubois rapproche d'ailleurs le genre du puzzle, et ajoute que s'est constituée une «mythologie du bricolage, du "do it yourself" ", qu'il appelle aussi "le mythe de la lecture active et participante ${ }^{8}$. Dans son ouvrage intitulé Mythologie du roman policier, Francis Lacassin aborde les mythes que sont devenus les grands personnages de détectives, comme Dupin, qui incarne «la raison contre les faits» ou Sherlock Holmes, "policier de laboratoire" ". On le voit dans ce bref parcours critique, le roman policier s'est bâti et cristallisé autour de mythes qu'on a tôt fait de confondre avec des caricatures - car «le mythe préfère travailler à

3. On pourra se reporter à la chronologie présentée par Fr. Lacassin, dans Mythologie du roman policier, Christian Bourgois, I993, p. II-22.

4. T. Todorov, "Typologie du roman policier", dans Poétique de la prose : choix, suivi de Nouvelles recherches sur le récit, Seuil, I980, p. II.

5. Ibid., p. I4. Voir également R. Baroni, La Tension narrative. Suspense, curiosité et surprise, Seuil, 2007, p. II4.

6. T. Todorov, «Typologie du roman policier», art. cité, p. I4.

7. Ibid., p. II.

8. J. Dubois, Le Roman policier ou la modernité, op. cit., p. 27.

9. Fr. Lacassin, Mythologie du roman policier, op. cit., p. 38 et p. 88 . 
l'aide d'images pauvres, incomplètes, où le sens est déjà bien dégraissé, tout prêt pour une signification : caricatures, pastiches, symboles, etc. ${ }^{\mathrm{I0}}{ }^{\prime}$.

Les mythes du roman policier dont nous voudrions traiter ici sont donc, d'une part, celui d'une narration uniquement utilitaire, au service des indices du code herméneutique ${ }^{\text {II }}$, et, d'autre part, celui de l'enquêteur. Or, les romans policiers de Fred Vargas mettent ces mythes à distance, en constituant un lieu de récupération et de transformation de ces derniers, non exempt d'une réflexion sur le genre policier, qui se rapproche d'une mythologie (en tant que discours sur le mythe). On essaiera donc de voir comment, sans aller jusqu’à la démystification, le roman policier chez Fred Vargas instaure une distance avec le réel indiciaire propre au genre, s'écrivant dans des marges oniriques qui deviennent le centre du roman.

Lanalyse s'appuiera sur trois romans policiers de Fred Vargas, L'Homme aux cercles bleus, Un peu plus loin sur la droite et Sous les vents de Neptuner2. Ces trois romans, à leur façon, mettent en scène des indices ou des coupables étranges, qui n’appartiennent pas forcément à la mythologie du policier : ce sont d'abord des cercles tracés à la craie bleue qui apparaissent sur les trottoirs, entourant des objets perdus qui déroutent le commissaire Adamsberg, c'est ensuite un bout d'os humain retrouvé dans une déjection canine sur une grille d'arbre parisien qui guide l'enquêteur Louis Kehlweiler jusqu'à un village breton, c'est enfin un assassin qui continue à commettre ses meurtres au trident par-delà la tombe et les océans.

Nous nous proposons de montrer d'abord que le roman policier de Fred Vargas obéit aux mythes du genre dans le traitement de l'objet comme partie prenante de l'enquête policière; cependant, l'intérêt du livre se déplace, et cette enquête n'est souvent qu'un prétexte à un roman qui fait la part belle aux incongruités et aux décalages, peignant des personnages qui gravitent dans le rêve plus que dans le réel, bien loin des règles utilitaires du roman policier. Pour finir, on étudiera spécifiquement deux cas, dans L'Homme aux cercles bleus et Un peu plus loin sur la droite, d'«indices» inutiles, dont on ne comprend pas le but, et qui remettent en cause toute la logique causale et indiciaire du roman policier, son mythe le plus ancré, pour ne pas dire le plus sacré.

IO. R. Barthes, Mythologies, op. cit., p. 200.

II. R. Barthes, $S / Z$, Seuil, I970, «Points», p. 26.

I2. L'Homme aux cercles bleus, J'ai lu, 1996 (désormais $H C B$ ); Un peu plus loin sur la droite, J'ai lu, 1996 (désormais UPL); Sous les vents de Neptune, Viviane Hamy, 2004 (désormais $S V N)$. Les références sont données dans le texte après les citations. 


\section{Le mythe de l'objet : de son utilité dans le roman policier}

Chez Vargas comme dans tout roman policier, les objets sont au service de l'enquête, et ils participent, dans le code herméneutique ou code de déchiffrement ${ }^{13}$, de ces éléments qui permettent de voir comment "une énigme se centre, se pose, se formule, puis se retarde et enfin se dévoile ${ }^{14}$ » : ce mythe de l'enquête policière, qui se bâtit à partir d'indices, de preuves, d'armes du crime et d'accessoires, est caractéristique du genre. Ainsi les objets chez Vargas peuvent être des armes du crime, que ce soit horloge ( $H C B$, p. I9) ou trident; mais ils sont aussi des informations et des preuves, qu'on trouve dans les journaux (p. 23), sur les lieux du crime, ou dans les maisons abandonnées ${ }^{15}$ par le meurtrier. Le lecteur familier des romans policiers construira, traditionnellement, ses déductions sur ces indices, activant des «scénarios intertextuels» et les développant hypothétiquement dans des "promenades inférentielles ${ }^{16}$ ».

Là où Vargas commence cependant à instaurer une distance avec le genre, entendons une distance parodique, un jeu avec le mythe de la preuve - jeu qui n'est donc pas, à proprement parler, une mythologie -, c'est lorsqu'elle fait affirmer lourdement l'insignifiance de l'indice, alors que ce dernier a pourtant beaucoup à signifier. De façon parodique, on affirme qu'il ne vaut rien, qu'il ne sert à rien : un commissaire à qui Kehlweiler montre le bout d'os humain trouvé sur la grille d'arbre lui dit : «c'est creux, c'est vide, c'est du vent» (UPL, p. 37). Ce bout d'os, trouvé dans «l'excrément dérisoire d'un chien inconséquent» (p. 13), singe donc la preuve habituelle : il est repoussant, semble inutile, et avec lui le mythe de l'indice est malmené. C'est à se demander si cette chose informe, "dont décidément personne n'avait l'air d'avoir quoi que ce soit à foutre» (p. IIs), cette chose trouvée dans une crotte de chien, n'a pas pour but de signifier la faillite du système indiciaire traditionnel, de signifier parodiquement que le roman policier se bâtit sur des absurdités, ou sur une réalité dont Vargas accentue les tentations du trivial. Si on n'est pas encore ici dans une réflexion sur le mythe de l'indice, comme on le verra à la fin de cette étude, on est déjà dans un jeu avec le mythe, dont on a conscience, et qu'on malmène.

13. Ch. Massol, Une poétique de l'énigme. Le récit herméneutique balzacien, Genève, Droz, 2006, p. 5 I.

I4. R. Barthes, $S / Z$, op. cit., p. 26.

15. Dans une grande maison alsacienne, Adamsberg remarque sur le sol une ligne plus claire, où la poussière est moins tombée : ce pourrait être la place du trident que conservait le meurtrier ( $S V N$, p. II7).

16. U. Eco, Lector in fabula, ou la coopération interprétative dans les textes narratifs, Grasset, 1985, p. 108 et p. I54. 
Cependant, les occurrences et les récurrences des indices et des preuves, qui servent l'enquête, sont faites mécaniquement, sans aucune irruption de l'incongruité ou de la fantaisie verbale qui disent la singularité des personnages.

\section{Un roman policier plus proche du rêve que du réel : le déplacement du topos policier}

On attend généralement des romans policiers qu'ils reproduisent avec attention la réalité, la société, le milieu du crime et celui des enquêteurs : «[d]ans sa tendance majeure, le texte policier s'inscrit dans la vaste configuration du discours réaliste», rappelle Jacques Dubois ${ }^{17}$. Or le monde des romans de Fred Vargas ne se veut pas réaliste. Ils ne tentent pas de signifier le langage du crime ou de la police; ils ont leur propre langage et créent leur propre monde, délibérément.

\section{Atmosphère onirique et réseaux-mana}

Le mythe ainsi se retourne ou s'inverse : ce qui semble une digression passe au premier plan, et la marche de l'enquête devient un prétexte pour cultiver la digression. On peut parler ici de déplacement de la priorité narrative sur des digressions oniriques. La ligne du récit perd en clarté et en simplicité, flottant dans un entre-deux qui penche plus vers le rêve que vers le réel.

Sous les vents de Neptune multiplie ces digressions; l'une des principales concerne les crapauds. Le commissaire Adamsberg raconte en effet dès le début du roman une anecdote de son enfance, sur les crapauds qui explosent lorsqu'on leur colle une cigarette au bec. Cette anecdote enchâssée prend place dans le récit d'un meurtre; or ce n'est pas à ce dernier que s'intéresse Danglard, pourtant capitaine de police :

- Pardon, coupa Danglard, soucieux. Le crapaud explosait-il réellement ou bien est-ce une image?

- Réellement. Il gonflait, il atteignait la taille d'un melon verdâtre et soudain, il explosait. Où en étais-je, Danglard? ( $S V N$, p. 5I)

Cette dernière question d'Adamsberg souligne bien la digression, de même qu'ensuite :

- Mais comment se fait-il que le crapaud fumait?

- Dites, Danglard, vous m'écoutez? Je vous raconte l'histoire d'un homme du

diable, et vous revenez sans cesse à ce satané crapaud.

I7. J. Dubois, Le Roman policier ou la modernité, op. cit., p. I22. 
- J'écoute bien entendu mais, tout de même, comment se fait-il que le crapaud fumait?

- C'était comme ça. Dès qu'on fourrait une cigarette allumée dans sa gueule, le crapaud se mettait à pomper. [...] Paf paf paf. Et soudain, il explosait. (Ibid., p. 52)

Cette matrice de l'anecdote des crapauds revient par bribes tout au long du roman. Les personnages trouvent un plaisir certain à répéter à tout propos l'expression "paf paf paf et explosion ${ }^{18}$ " : détachée de son contexte premier, elle prend alors une allure décalée, et devient une référence partagée par les héros et par les lecteurs, mais qui décourage les personnages simplement de passage dans le récit. Vargas renverse ici le mythe policier pour instituer une sorte de mythe propre à ses romans : ceux-ci s'adressent particulièrement aux lecteurs suffisamment amateurs du genre pour comprendre en quoi son écriture joue avec les règles, et qui apprécient en même temps ce décalage.

Ce procédé typiquement vargasien d'investissement d'une expression, ou plutôt d'une onomatopée, de tout un passif romanesque est encore à l'œuvre dans le dernier roman, paru en 2008, Un lieu incertain : alors que le commissaire Adamsberg demande très sérieusement à son compagnon de voyage, Vladislav, s'il a des ancêtres dont le nom commence par "Plog», le jeune homme, hilare, répète la syllabe à l'envi et l'utilise à tort et à travers : "Plog" devient un mot "dont le sens varie selon le contexte. Qui peut signifier "certes", "exactement", "d'accord", "compris", "trouvé", ou éventuellement "foutaises" 19 ". "Plog» est en quelque sorte institué "mot-mana», tel que le définissait Barthes, "un mot dont la signification ardente, multiforme, insaisissable et comme sacrée, donne l'illusion que par ce mot on peut répondre à tout ${ }^{20}$ ", "quelque chose comme une place vide où l'on dépose la collection entière des sens inavouables et que l'on sacralise comme un tabou ${ }^{21} »$. On pourrait ainsi dire que Vargas fonde ses romans sur certains mots ou réseaux-manas, qui reviennent dans des situations saugrenues, et contribuent à poétiser l'intrigue, la centrant sur ces digressions apparemment excentrées, irradiant pourtant l'ensemble de ses romans. Un lieu incertain voit ainsi fleurir en ses marges une sombre histoire d'ours blanc et de thékophages (des mangeurs d'armoires), et même l'histoire des crapauds est brièvement rappelée : les mythes vargasiens semblent donc investir le genre entier tel que l'auteur le conçoit, et non se renouveler à chaque roman.

18. $S V N$, p. 58, 63, 99, I23, I43, 248, ou 365 .

I9. F. Vargas, Un lieu incertain, Viviane Hamy, 2008, p. 319.

20. R. Barthes, Roland Barthes par Roland Barthes, dans CEures complètes, É. Marty (éd.), Seuil, I995, p. I94.

2I. R. Barthes, Mythologies, op. cit., p. I29. 
Au sein d'un même ouvrage, ces réseaux-mana se croisent et s'entremêlent volontiers : une autre récurrence onirique de Sous les vents de Neptune concerne les contes de fées, liant en cela les crapauds et les princes charmants. Le commandant Trabelmann affirme en effet au commissaire : "J'ai toujours adoré les contes.» (SVN, p. 75.) Adamsberg conclut au milieu du roman : "Décidément, il n'y avait pas que Trabelmann pour aimer les contes. Mordent aussi. Tout le monde peut-être, et même Brézillon» (p. 20I). Il est évidemment facile de lire ici l'ambition des romans de Fred Vargas, de raconter simplement une histoire qui prendrait l'allure d'un conte, malgré son aspect policier, car elle baignerait dans un univers moins réaliste que merveilleux, ménagé par ces digressions oniriques, dont on trouve encore d'autres exemples dans ce même roman avec la cathédrale de Strasbourg, à laquelle le commandant Trabelmann compare l'ego d'Adamsberg (p. 93). Dans ses rêveries, le commissaire vient au cours du roman encastrer fantasmatiquement tous les animaux possibles dans les fenêtres de ladite cathédrale; cela commence par le dragon des contes, dont il a parlé avec le commandant :

[...] il était en train de se demander si, en enfonçant un dragon tout entier dans le portail de la cathédrale de Strasbourg, celle-ci aspirerait et paf paf paf exploserait? (p. Ioo)

La digression sur les crapauds croise le dragon des contes et la cathédrale "égo-thique», selon le mot d'un personnage (p. 97), et ainsi tout se mélange et s'entrecoupe. À la fin du roman, Adamsberg adresse une pensée à la cathédrale de Strasbourg, métamorphosée par ses rêveries :

Adamsberg s'aperçut, en considérant la cathédrale, que la ménagerie avait déserté les lieux, clocher, fenêtres hautes, fenêtres basses et portail compris. Les bêtes avaient réintégré leurs lieux ordinaires, Nessie dans son loch, les dragons dans les contes, les labradors dans les fantasmes, le poisson dans son lac rose, le boss des bernaches sur l'Outaouais, le tiers du commandant dans son bureau. La cathédrale était à nouveau le pur joyau de l'art gothique s'élevant librement dans les nuages, bien plus haut que lui. (p. 436)

L'intrigue est donc le moment où les fils se lient, s'embrouillent, se perdent, comme les pensées du commissaire; quand elle se résout les rêveries prennent fin, et l'ordre du récit devient clair, comme les lignes de la cathédrale : on a là une métaphore de la conduite de l'enquête, ce mythe narratif dont on a parlé - mais, hypertrophiée, la métaphore prend le pas sur ce dernier.

Il y a, au-delà des réseaux singuliers et différents que ménage chaque roman, une récurrence plus personnelle que l'on pourrait noter dans les romans policiers de Fred Vargas : c'est la rêverie de l'eau. Le qualificatif "poissonneux", pour désigner un homme qui échappe à la compréhension, à la préhension ou à l'analyse, passe par exemple d'un roman à l'autre (UPL, 
p. I26 et $H C B$, p. 50). L'eau elle-même est surtout représentée comme une eau stagnante. Ce qu'un enquêteur appelle «l'état de flottaison du maire» de Port-Nicolas (UPL, p. 208), personnage souvent associé à une eau dormante, correspond à un état de flottaison de l'intrigue, pas encore résolue, dont on entrevoit la solution, et qui se perd dans des digressions liquides. Le réseau lexical est à nouveau métaphore de l'intrigue, qui flotte "entre deux eaux", pour citer encore Un peu plus loin sur la droite, entre le reflet et l'idée, le rêve et la réalité. Car l'eau apparaît aussi dans Sous les vents de Neptune, à travers le lac Pink, au Québec, auquel se rend Adamsberg. Les eaux de surface de ce lac ne se mêlent pas aux eaux du fond, qui sont fossilisées :

Le pire étant qu'un poisson marin y vivait encore, issu du temps où la mer était encore là. [...] Il eut beau relire le panneau, le poisson inconnu ne portait pas de nom. [...] Réchauffant ses mains, il recopia précisément le dessin de ce foutu poisson qui nageait entre ciel et enfer. (SVN, p. I54-I55)

Le poisson enfermé dans ces eaux n’a pas de nom : il représente ce à quoi on n'a pas accès, les indices de l'enquête qui se refusent et qui restent enfouis. Et si le poisson n'a pas de nom, c'est que l'obscurité et le rêve passent souvent au premier plan, reléguant l'intrigue au rang de prétexte. L'atmosphère onirique, voire merveilleuse, des romans de Vargas, ainsi que la récurrence de réseaux lexicaux, caractérisent tout aussi bien la marche de l'enquête que les enquêteurs.

\section{La caractérisation monodique ou monomaniaque des personnages}

Dans le roman policier, le personnage est généralement défini par quelques accessoires ou détails qui suffisent à lui donner consistance. Chez Agatha Christie par exemple, note André Vanoncini, «la figure du détective n’échappe pas à une stylisation extrême ${ }^{22}$ »; quant à Jacques Dubois, il écrit au sujet des personnages du roman policier : "[à] leurs "traits logiques" se superposent quelques détails "mythologiques" (la pipe de Rouletabille ou celle de Maigret) mais rien que de très rudimentaire et, somme toute, le minimum requis par les besoins de la représentation ${ }^{23}{ }^{\prime}$. Yves Reuter note lui aussi : "Privés, truands et vamps appartiennent désormais à notre mythologie ${ }^{24}$ » - si le roman policier se constitue bien sur des mythes, on voit ici que les personnages ne sont pas les moindres.

Ceux de Vargas se définissent aussi par des objets ou des détails qui reviennent de façon lancinante, comme pour les réseaux lexicaux dont nous

22. A. Vanoncini, Le Roman policier, Presses universitaires de France, 1993, p. 38.

23. J. Dubois, Le Roman policier ou la modernité, op. cit., p. 88.

24. Y. Reuter, Le Roman policier, Armand Colin, 2005, p. 7. 
avons déjà parlé, constituant pour chaque personnage un "univers» unique et particulier (de la vieille Marie, on dit en effet : "Personne n'aurait été lui prendre des bigorneaux, c'était son univers»-UPL, p. II3). Vargas obéit donc bien à ce mythe du roman policier lorsqu'elle attribue à Louis Kehlweiler, l'enquêteur d'Un peu plus loin sur la droite, une phrase qui scande le roman : "Je veux une bière ${ }^{25}$." Mais, on le voit, le mythe d'un personnage n'est pas ici constitué par un objet : il prend place, directement, dans le langage, même si les héros peuvent être rattachés à un élément récurrent, comme les griffonnages d'Adamsberg dans L'Homme aux cercles bleus ${ }^{26}$ ou le pompon rasé du bonnet de Danglard dans Sous les vents de Neptune.

Vargas, cependant, instaure une distance avec le mythe puisque ce type de caractérisation quasi unique - et lexical - des personnages, par ailleurs peu décrits, est mis à nu comme tel à l'intérieur du roman : le lieutenant Violette Retancourt est "capable, ainsi qu'elle l'avait exposé elle-même, de convertir son énergie à sa guise» ( $S V N$, p. 19) - cette description-définition court l'ensemble du roman ${ }^{27}$. Et lorsqu'on le fait remarquer à Adamsberg en lui disant "C'est un officier polyvalent qui convertit son énergie en ce qu'elle veut", le commissaire met au jour le mythe en répondant : "Oui, on sait cela. En moins d'un an, c'est presque devenu un mythe» (ibid., p. 2II). Même les personnages ont donc l'impression d'être définis par un seul trait, sans cesse répété dans le discours : on a là l'inscription intéressante, dans une description rituelle et mythique du personnage par une expression unique, d'une conscience de cette mythification à outrance. La voix du mythe coexiste, dans le discours des personnages, avec la voix de la démystification, ou tout au moins la conscience d'une mystification, ce qui est déjà mettre cette dernière à distance. Prenons un autre exemple de cette même conscience, un dialogue entre le commissaire et son adjoint : "Je commence à croire que Retancourt est une femme d'exception. Elle parvient à convertir son éner... / - Je sais, Danglard, je sais» (SVN, p. 2I2). Le commissaire interrompt le discours du mythe, trop bien connu; mais malgré sa défiance initiale à l'égard de cette caractérisation récurrente et monolithique, Adamsberg, comme le lecteur, finit par s'y habituer, et même à y adhérer, en se l'appropriant : «Elle convertit son énergie en ce qu' elle veut.» lui dit-on. Et il répond : "Je le sais. C'est mon lieutenant» (p. 274). Ainsi, si le mythe est mis à distance, il est finalement accepté comme tel par les personnages qui en avaient conscience.

Ces derniers exemples nous paraissent importants : ils esquissent un devenir des Mythologies qui serait non une démystification théorique ou théorisée,

25. UPL, p. I38, I53, I55, I64, I84, 213, 254.

26. HCB, p. I6, 22, 28, 30, 35, 36, 87, 91, 92, 93, 99, I0I, I39, I46, I73.

27. SVN, p. 35, 228, 240, 24I, 28I, 284. 
mais une conscience du mythe qui, si elle ne passe pas par un métalangage, un langage sur le mythe, permet de conserver avec celui-ci une distance qui se construit sur une dynamique dialogique, dans les deux voix croisées du mythe et de la conscience du mythe adoptées par les personnages, distance qui ne va pas jusqu'à la démystification - puisqu'elle signifierait l'abandon de la forme policière - mais qui permet de passer d'un mythe générique à un mythe personnel, inscrit dans l'écriture d'un auteur particulier.

Les personnages décrits à grands traits présentent tous, semble-t-il, la même caractéristique, qui appartient à cette mythologie personnelle de Vargas : à l'image de l'intrigue entre deux eaux, ils semblent aussi flotter entre rêve et réel, comme déplacés ou décalés dans celui-ci.

\section{Des personnages inadaptés au réel}

Les détails associés aux personnages vargasiens peuvent sembler insolites ou étranges. Par ailleurs, contrairement à ceux qui sont devenus mythiques (parfois tout à fait faussement, comme conséquence de la caricature et de ses déformations), du violon de Sherlock Holmes aux chaussures cirées d'Hercule Poirot, ils ont moins tendance à faire du héros un super-héros (dans la réflexion ou dans l'action) qu'un anti-héros; personnage inadapté au réel, le héros vargasien n'est adapté qu'au rêve et au langage au sein duquel il se constitue, et qui n'a pas vocation à signifier le réel, mais à en inventer un, qui a ses codes. L'usage que les enquêteurs font des objets de la vie quotidienne n'est pas celle qu'on en attend; il devient étrange, bizarre, détourné, excentré, de même que l'est l'intrigue, dans ses digressions.

Ainsi, alors que Louis Kehlweiler boit ses bières, Marc, son assistant, fait tout autre chose de sa bouteille : il commence par regarder au fond, à y coller son oil; puis il y coince son doigt ${ }^{28}$, qu'il n'arrive pas à sortir, si bien qu'après un épisode à forte tension dramatique, pendant lequel il est resté le doigt dans le goulot, Louis lui fait remarquer : "N'empêche que cela fait une heure que tu as le doigt coincé dans le goulot de cette bouteille et que tu ne peux plus le sortir. C'est stupide, mais il faudrait intervenir." (UPL, p. 227.) L'aspect dérisoire et «stupide» de l'usage que Marc a fait de sa bouteille est un exemple typique de ces rêveurs qui peuplent les romans de Fred Vargas - on préfèrera ici le terme rêveurs à celui de marginaux : s'il a pu être utilisé lors d'un colloque à Cerisy ( $\mathrm{I}^{\mathrm{er}}$-II août 2007) intitulé "la fiction policière aujourd'huii ${ }^{29}$ ", il nous semble que, l'univers de Vargas étant peuplé de

28. UPL, p. I4I, 2I4, 216, 218.

29. L'intervention de L. Sudret se nommait «Du traitement de la marginalité dans les romans policiers de Fred Vargas»; on mentionne en bibliographie une édition de L'Homme 
"marginaux", ceux-ci ne le sont guère que dans notre univers, et non dans celui de la fiction, où ils sont à l'aise (même si ce n'est pas financièrement).

Le personnage le plus rêveur et le plus décalé reste le commissaire Adamsberg; l'une de ses collègues l'avait décrit de la sorte dans L'Homme aux cercles bleus : "tu es là, tu traînes, tu rêves, tu contemples les murs, tu griffonnes des croquis sur tes genoux" (HCB, p. II). Les errements, réels et métaphoriques, supposés par les deux verbes traîner et rêver sont très représentatifs du personnage, constamment défini par cette inadaptation au réel : lui-même dit à un homologue québécois dans Sous Les Vents de Neptune : «je marche, je déambule, je rêve». Ce à quoi son collègue, dans son parler national, réplique : "Tu résous tes affaires en pelletant des nuages?» ( $S V N$, p. I66). C'est assez pour qu'Adamsberg devienne un "pelleteux de nuages ${ }^{3 \circ}$ » dans le reste du roman, voire dans les suivants. Mais c'est à son adjoint Danglard qu'il revient de définir le plus justement ses digressions oniriques : «Et seul Adamsberg était capable de distordre la vie ordinaire pour en extraire ces incartades, ces courts éclats de beauté saugrenue», "comme si, chez lui, la limite entre rêve et réel n'était pas très marquée» (ibid., p. 28, 209). On relève aisément ici cet entre-deux dont on a déjà parlé, «entre rêve et réel ", ou entre «vie ordinaire» et vie onirique.

Fred Vargas, enfin, joue du décalage de ses personnages pour parodier les héros traditionnels. Ainsi, dans sa première aventure, Adamsberg n'a pas de montre (HCB, p. I3), mais il en a deux sur le même poignet dans Sous les vents de Neptune ( $S V N$, p. II6), preuve qu'il ne fait rien comme les autres. Adamsberg reste cependant un policier, et à ce titre il lui faut quand même se préoccuper de l'heure, piéger les suspects en "surveillant ses montres» (ibid., p. 39I). Ces deux montres ont tout à la fois le rôle de souligner l'originalité du commissaire, en même temps qu'elles constituent, par leur multiplication, une parodie d'un des mythes du roman policier, où le héros, homme d'action et homme pressé, ne cesse de consulter sa montre.

Cependant, si elle parodie le mythe policier, Vargas ne l'abandonne pas pour autant : ses romans mettent en question l'utilité narrative et fonctionnelle du genre sans cependant le démystifier.

aux cercles bleus établie par ses soins pour les classes de collège et lycée professionnel (Magnard, 2006) : dans l'appareil pédagogique elle note que "presque tous les personnages sont des marginaux qui ne réussissent pas à s'adapter au moule conformiste de la société» (p. 233).

30. SVN, p. I70, 435 et 442 . 
Recherches \& Travaux $-\mathrm{n}^{\circ} 77$

\section{Réflexion autour de l'inutile, ou l'amorce d'une démystification du roman policier}

Chez Vargas, on l'a vu, les objets sont, comme on l'attend d'eux dans les romans policiers, des moteurs de l'intrigue, des accessoires utiles ou des preuves. On a pourtant montré que nombre de détails n'avaient aucun rôle dans l'intrigue, mais permettaient de cimenter une structure reposant sur la récurrence et l'incongruité poétique et onirique. D'autres objets paraissent ne servir à rien, comme les cercles bleus tracés sur les trottoirs, dont on ne comprend pas le but, comme la Machine à rien dans Un peu plus loin sur la droite.

\section{Les cercles bleus}

Dès l'apparition des cercles bleus dans les rues parisiennes, les enquêteurs se demandent à quoi peuvent bien rimer ces cercles à la craie qui entourent des objets perdus sur le trottoir, et les hypothèses se multiplient : la cause, le mobile, sont interrogés, surtout par les policiers, qui réfléchissent évidemment en termes de causalité : "Pourquoi de si grands cercles pour de si petites choses? se demanda Danglard. Il ne s'était pas jusqu'ici avisé de cette disproportion.» (HCB, p. 4I.) Ainsi, par la multiplication dans le roman des interrogations et des hypothèses, des journalistes et des psychiatres, sur la motivation du "cercleur", se pose la question de l'inutilité, d'une faille dans la logique policière, faille que serait l'absence de motif, le ridicule, l'incompréhensible.

On trouve aux pages 24 à 26 du roman un assez long article de journal sur les cercles bleus, que le commissaire découvre en même temps que le lecteur. Cet article fait la liste des objets (une soixantaine) déjà cerclés. Elle commence par «douze capsules de bière, une cagette de légumes, quatre trombones, deux chaussures, une revue, un sac en cuir, quatre briquets, un mouchoir, une patte de pigeon" - arrêtons-nous là, l'article parlant d' "énumération fastidieuse", et soulignant l'aspect incongru et énigmatique de cet acte de cerclage en même temps que l'absence de liens entre les objets cerclés. D'ailleurs, l'article finit par :

C'est parce que le psychiatre René Vercors-Laury s'est intéressé aussitôt à ce cas en tentant d'y apporter ses lumières, que l'on parle maintenant de «l'objet revisité» $[\ldots]$. Chacun cherche sans trouver quelle peut bien être la pulsion qui anime l'homme aux cercles bleus. (HCB, p. 25-26) 
Ainsi l'absurdité, la faille causale, sont trop angoissantes pour être laissées à l'incompréhension, à l'absence de motivation : il faut théoriser, comprendre la démarche de l'homme aux cercles bleus, y voir un acte politique. Adamsberg rend visite, plus loin, au psychiatre mentionné, et sa théorisation s'étend sur plusieurs pages; nous pouvons en citer ce passage, tout à fait significatif du discours qu'on tient sur le réel pour tenter de lui donner un sens, un but, une cause : le psychiatre affirme donc que :

[c]et homme semble aux prises avec un douloureux questionnement, métaphysique, philosophique ou pourquoi pas poétique, sur la manière dont l'être humain choisit de faire commencer et cesser la réalité des choses, dont il s'en pose l'arbitre, alors qu'à ses yeux, peut-être, la présence des choses continue hors de nous [...]. (Ibid., p. 45)

Le fait de dévider les grands adjectifs "métaphysique», "philosophique» et "poétique» est une manière d'investir de sens quelque chose qui en semble dépourvu, par crainte de reconnaître son absence même. De la même façon, un autre personnage parle des cercles bleus en termes de «reconnaissance métaphorique des trottoirs comme nouveau champ de science» (ibid., p. II6). Ces interprétations soulignent, semble-t-il, la manie causale et motivatrice propre au roman policier; ce métalangage sur les cercles bleus, inscrit dans le récit, instaure là encore une distance avec le mythe, en invitant à réfléchir sur l'absence de motif ou de mobile. Le roman met ainsi en scène dans le même geste la tentation mythique d'ordonner le monde et de l'expliquer entièrement, comme on le fait dans le roman policier, et la remise en cause de la causalité, par l'irruption dans le réel policier, efficace, de bribes in-signifiantes, qui lui rendent un peu de son opacité et de son mystère.

Mais l'enquête policière, c'est là l'un de ses mythes, se doit finalement d'ordonner un monde en pertes de repères et de sens, $d^{\prime}$ ' organise[r] un monde sans contradictions parce que sans profondeur": le mythe en effet "fonde une clarté heureuse ${ }^{3 \mathrm{I}} »$. On finira donc par découvrir que les cercles bleus avaient bien un rôle : ils devaient accueillir des cadavres, cacher un crime en particulier dans une série macabre. Alors, certes, le mythe a été mis en question, mais on n'a pas vu s'opérer pour autant une entière démystification : la mythologie du genre policier l'emporte, celle d'un réel d'abord obscur, mais toujours heureusement clair à la fin. Il reste ces cercles qui ont quand même évoqué, pendant tout le roman, la menace de l'absurde ou l'inquiétude de l'insignifiant, et, plus encore, se jouant des mythes du genre, celle de l'inutile. C'est cette tentation de l'inutile, paradoxale pour cette forme littéraire, qu'on retrouve encore dans Un peu plus loin sur la droite, avec la Machine à rien.

3I. R. Barthes, Mythologies, op. cit., p. 217. 


\section{La Machine à rien}

Cette Machine à rien ne se manifeste que dans la seconde moitié du roman. Marc, l'assistant de l'enquêteur, raconte comment il l'a découverte :

[...] il avait trouvé là, en face du camping, en face de la grande surface commerciale, un truc hallucinant. Une espèce de machine de quatre mètres de hauteur, une immense et magnifique masse de ferraille et de cuivre, organisée dans ses plus petits détails, bourrée de leviers, d'engrenages, de disques, de pistons, et le tout ne servant strictement à rien. (UPL, p. 156)

On voit ici comment l'adverbe «rien» est retardé, constituant la révélation finale de la longue phrase. L'adjectif «hallucinant» dit bien le scandale d'une machine inutile dans un univers réel, celui du supermarché et du camping, où tout doit être utile (la consommation a ses mythes, elle aussi), et dans un genre où le moindre indice est signifiant. La machine cependant a un rôle, mais un rôle idiot : on lui pose une question, et en activant une manivelle, elle imprime une phrase, qui tient lieu de réponse divine. Elle ne produit donc rien sinon une réponse aléatoire, parodie grimaçante du dialogue avec Dieu; contrairement aux réponses claires et éclairantes d'une enquête policière, la Machine à rien ne fournit que des propos absurdes.

Cette "fabuleuse Machine à rien" (ibid., p. I63), que son créateur appelle "énorme machine cinglée», ou "usine à questions insatisfaites» (p. I87, I88), connaît elle aussi le démon de la théorisation : cette fois-ci, il s'empare du créateur de la machine lui-même, qui explique ce qu'il voulait en l'inventant : "Construire, mécaniser l'inutile. [...] Gloire au fonctionnement, et gloire au dérisoire et à l'inutile!» (p. I87.) L'expression "Machine à rien" apparaît après cette théorisation (p. 204). Le créateur de la Machine à rien fait finalement la même chose que l'homme aux cercles bleus : il bâtit «ce monument de l'inutile» (p. 240), dont on parle, qu'on observe, et qui pose la question de sa fonction, ou plutôt de son absence de fonction.

Cependant, puisqu'on est malgré tout et finalement dans un roman policier, l'inutilité ne se pose pas comme une angoisse, une démystification, seulement comme une question, un détournement du mythe. Car la Machine à rien est à l'image du roman policier de Fred Vargas : on parle pour ne rien dire d'utile (à la marche de l'enquête), on construit pour ne rien faire, pour le plaisir de parler ou de construire; on relègue l'utilité, donc l'intrigue policière, au second plan, on la malmène. Mais finalement, on la récupère à la fin, lorsqu'on montre que ces éléments absurdes, les cercles bleus comme la Machine à rien, cachent en réalité une autre forme d'utilité, criminelle : les cercles bleus permettent au meurtrier de perdre son crime au milieu d'une série qui paraît l'œuvre d'un maniaque, la Machine à rien a été construite sur 
la tombe d'une des victimes de son créateur, pour qu'on ne découvre jamais qu'il est un meurtrier - les cercles comme la machine cachent donc des crimes, de la façon la plus utilitaire qui soit. Si bien que Marc conclut :

- Le rectangle tombal est coincé sous la Machine à rien, je te dis. Machine à rien, mon œil. Ça m’étonnait aussi qu'un gars comme Sevran se soit crevé pour zéro, ce n'est pas son profil. Avec l'ingénieur, il faut que tout serve. Je sens les gars qui ont le goût de l'inutile, on repère toujours ses pareils. Lui, il a le sens exaspéré de l'utile. Alors, sa machine, elle sert diablement bien à quelque chose. À coincer la tombe de Diego, deux pieds de fer par-dessus et on n'y touche plus. (HCB, p. 237)

S'il y a donc des personnages, chez Vargas, qui ont le goût de l'inutile, qui coincent leurs doigts dans les goulots, qui se préoccupent des crapauds qui explosent ou qui portent deux montres, ceux qui obéissent le plus à la logique de l'utile, de l'efficace, de la logique, ce sont les meurtriers, ne serait-ce que pour que l'intrigue policière puisse exister. Et à l'image du déplacement du centre de l'intrigue sur des éléments incongrus, oniriques, ces éléments inutiles, cercles et machine, déplacent le roman policier dans des zones frontières. Certes, on récupère l'intrigue, l'utilité, à la fin, quand on a démontré que ces machines à rien avaient une raison d'être. Mais de la même façon que la Machine à rien donne des réponses fausses ou ridicules, incohérentes, toutes faites (à l'image d'un prêt-à-lire, d'un mythe du roman policier qu'on verserait éternellement dans le même moule), le roman résout une intrigue toute faite, dont la valeur ne réside vraiment que dans les marges oniriques et absurdes, in-signifiantes.

Ainsi, l'un des nombreux devenirs des Mythologies serait ce roman policier qui instaure avec son genre une distance souvent parodique n'allant pas jusqu’à la démystification, un roman qui s'écrirait dans ses marges, où l'intrigue, reléguée au second plan, cèderait le pas aux digressions et aux détours oniriques.

Certes le réel sert l'intrigue policière, dans la mesure où il fournit des armes, des preuves, des indices. Mais ces indices peuvent être parodiques, souligner la tentation du trivial que connaît le genre, la conscience qu'on a des mythes qui le gouvernent. Le réel, chez Vargas, est distordu, malmené : le mythe du roman policier se déplace du réel au rêve, mettant au premier plan des indices lexicaux plus que criminels, prenant l'intrigue policière comme prétexte pour permettre à des rêveries et à des rêveurs de s'épanouir dans un univers poétique, loin du mythe de l'enquêteur traditionnel. Finalement, en étudiant deux cas précis de tentations de l'inutile au sein d'une forme littéraire qui ne se nourrit que de l'utile, indiciaire et narratif, on a montré 
qu'on ne peut pas aller jusqu'à parler de démystification du genre chez Vargas puisque le langage de la démystification est lui-même mis à distance : dans L'Homme aux cercles bleus, tenter de combler par le métalangage l'insupportable faille du réel n'est qu'une mystification, dans Un peu plus loin sur la droite c'est également un mensonge, puisque cercles et machine ont une utilité criminelle. En revanche, la coexistence constante de deux voix, celle du mythe et celle de la conscience du mythe, conscience qui passe par le discours qu'on en tient, par la distance qu'on instaure avec lui, constitue une réflexion sur le genre. Vargas n'en dépasse jamais les limites, car elle écrit avant tout des romans policiers : le flottement entre le réel policier et un réel incongru, distordu, inutile, est à l'image de l'hésitation entre le genre et sa démystification, jamais complètement atteinte, dont on se méfie même - et cette phrase de Barthes fait bien écho à cette hésitation : «nous voguons sans cesse entre l'objet et sa démystification, impuissants à rendre sa totalitééc ${ }^{22}$.

Parodiant et mettant à distance les mythes du roman policier, Vargas institue ainsi les siens, qui font tout le charme de ses romans, mais qui finissent peut-être, ou finiront, par sentir la recette. Un lieu incertain a remporté en 2008 un beau succès de librairie : il peut cependant paraître moins abouti, en ce qu'il renouvelle assez peu la mythologie propre à chaque roman, récupérant essentiellement celle des précédents; les personnages vivent en vase clos, et les mythes tournent sur eux-mêmes. Si Vargas cimente ainsi davantage la communauté de ses lecteurs (dont on sait la fidélité et l'enthousiasme), par le jeu de la reconnaissance et du déjà-vu, elle n'oxygène guère l'univers de ses personnages, quand bien même ils parcourent l'Europe. Alors, certes, Vargas institue ses propres mythes, et Fioretto peut se moquer de ces romans où «les assassins étaient agrégés d'histoire et de lettres classiques» ou "serial killers médiévistes", et où parlent "les gens de peu tels que les imaginent les universitaires qui ont lu Bourdieu ${ }^{33}$ "; ce sont effectivement des mythes vargasiens qui font le charme de ces romans. Mais on regretterait que ces mythes ne soient plus, comme auparavant, renouvelés, mis à distance, et malmenés à leur tour. Car tout le succès des romans policiers de Fred Vargas nous semble reposer sur ce fragile équilibre entre une voix familière et des incongruités renouvelées, qui fascinent le lecteur comme Marc l'est par la Machine à rien, ou Mathilde par l'homme aux cercles bleus. La fascination vient de ces différents enquêteurs qui, comme le commissaire Adamsberg, sont avant tout des rêveurs - et les assassins ne le sont pas, ou bien moins, tout en prétendant l'être. Dans l'univers romanesque vargasien peuplé de rêveurs, c'est l'assassin,

32. Ibid., p. 233.

33. P. Fioretto, Et si c'était niais? Pastiches, Chiflet \& Cie, 2007, p. 39, 40 et 44. 
avec son sens de l'utile, qui devient le marginal. Par un étrange renversement, l'homme qui réussit (l'assassin chez Vargas est volontiers juge, ingénieur, médecin, professeur émérite), celui qui a le goût des détails et de l'utile, c'est celui qui fait scandale dans l'univers de la fiction, celui qui trouble les digressions, parce qu'il tue au lieu de rêver, de lire et de parler. 